\title{
ORBITAL STRUCTURE OF THE TWO FIXED CENTRES PROBLEM
}

\section{A. CORDERO}

Dpt. Matematica Aplicada. Universidad Politécnica de Valencia. Valencia. Spain, e-mail: acordero@mat.upv.es

\section{J. MARTINEZ ALFARO}

Dpt. Matemdtica Aplicada. Facultat de Matematiques. Universitat de Valencia. Valencia. Spain.e-mail: Jose.Mtnez.Alfaro@uv.es

and

\section{P. VINDEL}

Dpt.Matemàtiques.Universitat Jaume I. Castello.Spain.e-mail: vindel@nuvol.uji.es

The set of orbits of the Two Fixed Centres problem has been known for a long time (Charlier, 1902, 1907; Pars, 1965), since it is an integrable Hamiltonian system.

We consider a plane that contains the fixed masses. Denote by $\varphi$ the angle defined by this plane and the one that contains also the third body. The momentum $p_{\varphi}$ is a first integral of the system and when $p_{\varphi}$ is different from zero, the manifold generated by the generalized coordinates and momenta are two copies of the threedimensional sphere $S^{3}$. If $p_{\varphi}=0$, that is to say when the planet crosses the line joining both suns, the motion is restricted to a planar one. All the equilibrium points appears in this case and therefore the phase spaces are more complex. We restrict our attention to this case which has two degrees of freedom.

It is again a Bott-integrable Hamiltonian system. The set of periodic orbits of this systems can be studied from a subset of them, the Non-Singular MorseSmale type orbits (see Casasayas, 1992). It is proved in Campos (1997) that a small perturbation of a Bott-integrable Hamiltonian system transforms it into a Non-Singular Morse-Smale system. The NMS periodic orbits belong to both the NMS system and the Hamiltonian one. Moreover, The NMS p.o. can be continued to nearly Hamiltonian systems. For instance, in our case to the Restricted Three Body Problem and in the study of the motion of a material point moving inside the gravitational field generated by two stars. This approximation is also useful when the motion of an artificial satellite around a spheroidal body is considered.

If $p_{\varphi} \neq 0$ the link generated by the NMS p.o. is the Hopf link. The orbital structure of the NMS type periodic orbits in the different cases of negative and positive energy is obtained (see Cordero et al., 1997b). We analyze the dynamical and physical consequences of this structure.

In the physical space it is shown (see Cordero et al., 1997a) that different regions of motion are delimited by limit curves corresponding to fixed points of the new Hamiltonian functions, all the possible orbits being classified. So, these NMS orbits become collision or limit orbits in the physical space.

As the Three Dimensional Two Fixed Points problem is considered an integrable approximation of the Three-Body Restricted problem, we study the equilibrium 
points of this system that come from the previous one, and it is found that in this case the Hamiltonian is not separable in two new Hamiltonian functions. At this point, we deduce from the NMS type orbits found in the Planar Two Fixed Centres Problem the behaviour of this quasi-integrable problem. The NMS orbits that we are using as an approximation to the Restricted Three Body problem are defined by certain equilibrium points on the separated phases spaces. Five NMS orbits appeared associated to other five equilibrium points: three of them are collision orbits and the other two are limit orbits.

Working with the equations of motion in hyperbolic coordinates and substituting the conditions of the NMS orbits corresponding to collision we obtain two equilibrium points in the restricted problem, i.e., this coordinate transformation has regularized the problem, removing the singularities.

\section{References}

Campos, B., J. Martínez Alfaro and P. Vindel: 1996, Orbital Structure in the Two Fixed Centres Problem, Proceedings of the Third International Workshop on Positional Astronomy and Celestial Mechanics, pp. 313-323.

Campos, B: 1997, Orbitas periódicas Anudadas en oscilaciones no lineales, Tesis Doctoral, Universitat Jaume I

Charlier, C.V.L.: 1902 Die Mechanik des Himmels, Volume 1. W. de Gruyter and Co, Volume 1, 1902; Volume 2, 1907.

Charlier, C.V.L.: 1907 Die Mechanik des Himmels, Volume 2. W. de Gruyter and Co

Cordero, A., J. Martínez Alfaro and P. Vindel: 1997a, Study of the orbits in the Two Fixed Centres Problem, Proceedings of the Forth International Workshop on Positional Astronomy and Celestial Mechanics, Peñiscola.

Cordero, A., J. Martínez Alfaro and P. Vindel: 1997b, NMS Periodic Orbits of the Two Fixed Centres Problem, Proceedings of the Forth International Workshop on Positional Astronomy and Celestial Mechanics, Peñiscola

Cordero, A.: 1997, Estudio analitico y geométrico del problema de los Dos Centros Fijos, Tesis de Licenciatura, Universidad Jaime I de Castellón

Pars, L.A.: 1965, A Treatise on Analytical Dynamics. Ed. Heinemann 